\title{
Blue-Emitting CdSe Nanoplatelets Enabled by Sulfur-Alloyed Heterostructures for Light-Emitting Diodes with Low Turn-on Voltage
}

\author{
Merve İzmir, Ashma Sharma, Sushant Shendre, Emek Goksu Durmusoglu, Vijay Kumar Sharma, \\ Farzan Shabani, Hamed Dehghanpour Baruj, Savas Delikanli, Manoj Sharma, and Hilmi Volkan Demir*
}

Cite This: ACS Appl. Nano Mater. 2022, 5, 1367-1376

Read Online

ABSTRACT: Colloidal nanoplatelets (NPLs) have emerged as the last class of semiconductor nanocrystals for their potential optoelectronic applications. The heterostructures of these nanocrystals can achieve high photoluminescence quantum yield and enhanced photostability, along with color purity. Such advantages make them a promising candidate for solution-processable lightemitting diodes (LEDs). However, to date, blue-emitting CdSe nanoplatelets (NPLs) exhibit poor photoluminescence quantum yield and also typically suffer from a rolled-up morphology. To mitigate these problems in this work, we propose and demonstrate efficient alloyed $4 \mathrm{ML} \mathrm{CdSe}_{1-x} \mathrm{~S}_{x}$ nanoplatelets having a CdS crown with enhanced photoluminescence quantum yields (up to 60\%) in the blue region $(462-487 \mathrm{~nm})$. We successfully used these NPLs as an electrically driven active emitter in the blue-emitting NPL-LEDs with a low turn-on voltage of $\sim 4 \mathrm{~V}$. The Commission Internationale de L'Eclairage (CIE)

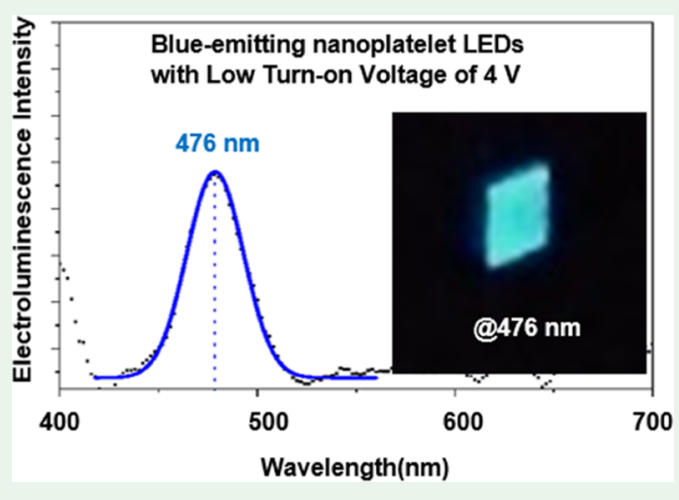
coordinates of $(0.23,0.14)$ were obtained for these blue-emitting NPL-LEDs. These emitters could potentially open up the opportunity for full-color displays using these NPL-based blue LEDs in conjunction with the red and green ones.

KEYWORDS: colloidal nanoplatelets, alloying, heterostructures, electroluminescence, light-emitting diodes, blue emission

\section{INTRODUCTION}

In the last decade, atomically flat semiconductor nanocrystals, commonly known as nanoplatelets (NPLs), have been introduced as a new class of solution-processed quasi-2D nanocrystals for the next-generation photonic applications. NPLs exhibit narrow emission bandwidth, giant oscillator strength, large absorption coefficient, and reduced Auger recombination. ${ }^{1-3}$ Such distinct properties make them attractive for optoelectronic devices including solar cells, ${ }^{4}$ luminescent solar concentrators (LSCs), ${ }^{5}$ lasers, ${ }^{6}$ and lightemitting diodes (LEDs). ${ }^{7}$

NPLs with different vertical thicknesses show discrete emission and absorption profiles independent of their lateral size. ${ }^{8,9}$ CdSe-based NPLs with three, four, and five monolayers (MLs) of vertical thicknesses terminated by Cd atoms on both sides exhibit emission peaks at $\sim 460,513$, and $550 \mathrm{~nm}$, respectively. ${ }^{10} \mathrm{~A}$ finer tuning of the emission peaks can be obtained with a combination of techniques such as thickness control, ${ }^{11}$ shell growth, ${ }^{12}$ and alloying. ${ }^{13}$ Doping also creates additional energy levels in the band gap of the host semiconductor NPLs, making Stokes-shifted emission possible. $^{14-16}$

Previously, synthesis of these CdSe-based NPLs with various heterostructures such as core-shell, core-crown, and core- crown-shell have been investigated to improve the photoluminescence (PL) quantum yield (QY) and their stabilities. ${ }^{6,17}$ Especially, shell growth of NPLs has been exploited using two different approaches of colloidal atomic layer deposition (c-ALD) and hot-injection shell growth techniques. With these methods, shell layers are vertically grown and the excitonic transitions are red-shifted in their heterostructures. The relaxation of quantum confinement results in such redshifting of the resulting excitonic features. ${ }^{18}$ In addition, to enhance the excitonic features of semiconductor nanocrystals, postsynthetic treatment processes such as doping can also be used. $^{19,20}$ Also, small amounts of doping can lead to the passivation of surface defects and can improve the overall excitonic properties.

In the family of colloidal NPLs, $3 \mathrm{ML}$ CdSe NPLs emit in the blue region at $\sim 460 \mathrm{~nm} .^{21}$ The PL QE of these thinner NPLs is reported to be around $5-10 \% .^{21,22}$ In addition, these

Received: November 18, 2021

Accepted: December 14, 2021

Published: December 28, 2021 


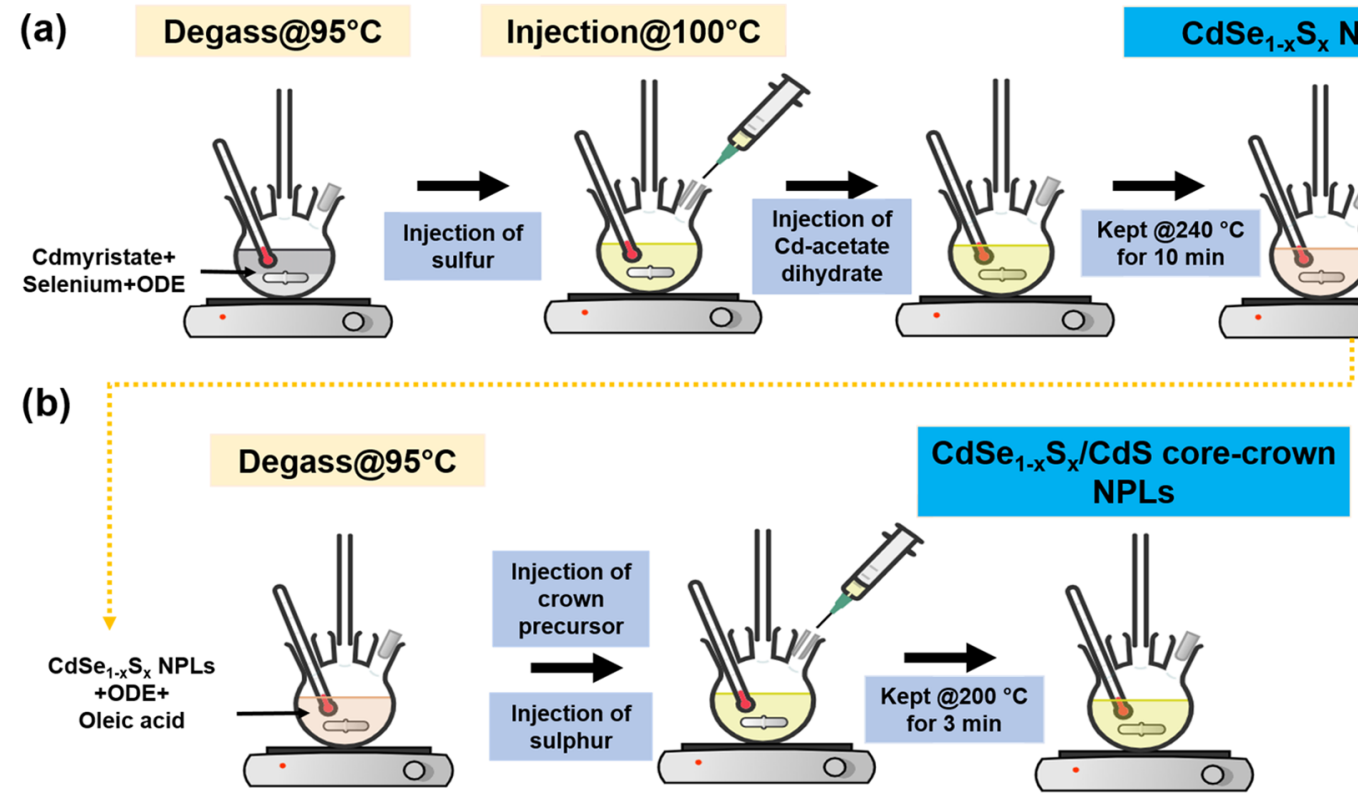

Figure 1. Synthesis of (a) alloyed $4 \mathrm{ML} \mathrm{CdSe} e_{1-x} \mathrm{~S}_{x}$ core NPLs and (b) $4 \mathrm{ML} \mathrm{CdSe} e_{1-x} \mathrm{~S}_{x} / \mathrm{CdS}$ core-crown NPLs.

NPLs tend to roll up with large lateral dimensions. Lasing in the blue range was reported with an amplified spontaneous emission (ASE) threshold of $\sim 50 \mu \mathrm{J} / \mathrm{cm}^{2}$, which is lower as compared to blue-emitting quantum dots. ${ }^{8}$ Additionally, amplified spontaneous emission (ASE) thresholds of $\sim 75$ $\mu \mathrm{J} / \mathrm{cm}^{2}$ were reported with $\mathrm{CdS} / \mathrm{ZnS}$ core/shell NPLs. ${ }^{23}$ Previously, our research group has also reported the ASE threshold of $\sim 2.7 \mu \mathrm{J} / \mathrm{cm}^{2}$ and a large net modal gain coefficient of $360 \mathrm{~cm}^{-1}$ in the blue region $(\sim 455-465 \mathrm{~nm})$ with $3 \mathrm{ML} \mathrm{CdS} / \mathrm{CdSe}$ core/crown NPLs. ${ }^{24}$ These findings indicate that the synthesis of engineered heterostructured NPLs can pave the way for the usage of NPLs for lasing and LED applications. Furthermore, Di Giacomo et al. have recently shown that cadmium carboxylate precursors with different chain lengths make it possible to tune the width and aspect ratio of $3 \mathrm{ML}$ CdSe NPLs with enhanced optical properties. $^{25}$ Additionally, to obtain the blue emission from these CdSe-based NPLs, alloying in the host nanocrystals also provides another opportunity to tune the excitonic properties. ${ }^{26}$ Fan et al. reported the successful tuning of the emission wavelengths from 520 to $481 \mathrm{~nm}$ by alloying sulfur in host CdSe NPLs (e.g., $\left.\mathrm{CdSe}_{1-x} \mathrm{~S}_{x} \mathrm{NPLs}\right){ }^{13}$ Moreover, earlier our group showed that alloyed $\mathrm{CdSe} \mathrm{S}_{1-x}$ core/shell NPLs possess a tunable emission in the range of 560-650 nm. In addition, these alloyed $\mathrm{CdSe}_{x} \mathrm{~S}_{1-x}$ core/crown NPLs exhibit an emission peak between $\sim 500$ and $\sim 540 \mathrm{~nm}^{27}$

Colloidally synthesized CdSe-based NPLs are promising next-generation low-cost and high-efficiency LEDs because of their outstanding properties. In early reports, Chen et al. demonstrated red NPL-LEDs using core/shell CdSe/CdZnS NPLs with the optimizations for achieving LEDs with color purity. ${ }^{7}$ Our group has recently reported the NPL-LEDs with saturated red emission using $\mathrm{CdSe} / \mathrm{Cd}_{0.25} \mathrm{Zn}_{0.75} \mathrm{~S}$ core/shell NPLs synthesized using hot-injection shell growth method (HIS) with a record external quantum efficiency (EQE) of $19.2 \%{ }^{28}$ In another report, green emission using $\mathrm{CdSe} / \mathrm{CdS}$ NPLs core/shell heterostructures was demonstrated to have an EQE of $5.0 \% .^{29}$ Also, the EL spectrum in green was reported with the narrow full width at half-maximum (FWHM) of 12-
$14 \mathrm{~nm}$ in CdSe/CdS core/shell NPL-based LED devices. ${ }^{29} \mathrm{~A}$ full-color display requires red, green, and blue emissions with high stability, efficiency, and color purity. However, to date, there has been only one report related to LED devices with cyan-emitting NPLs. ${ }^{13}$ This could be because of the rolled-up morphology of blue-emitting $3 \mathrm{ML}$ CdSe NPLs with poor optical properties. ${ }^{30}$

Herein, for the first time, we show efficient blue-emitting NPLs using alloyed $\mathrm{CdSe}_{1-x} \mathrm{~S}_{x} / \mathrm{CdS}$ core-crown heterostructures, emitting at $\sim 462-487 \mathrm{~nm}$, with a PL-QY as high as $\sim 60 \%$. Also, we demonstrate blue NPL-LEDs with an FWHM of $34 \mathrm{~nm}$ possessing an EL spectrum peaking at $\sim 476 \mathrm{~nm}$ and the Commission Internationale de L'Eclairage (CIE) coordinates of $(0.23,0.14)$ and a low turn-on voltage of $\sim 4 \mathrm{~V}$. Our findings indicate that the further development of blue-emitting NPL-LEDs will add to the NPL-based red, green, and blue LED family for full-color display and lighting applications.

\section{MATERIALS AND METHODS}

2.1. Synthesis. 2.1.1. Chemicals. Cadmium nitrate tetrahydrate $\left(\mathrm{Cd}\left(\mathrm{NO}_{3}\right)_{2} \cdot 4 \mathrm{H}_{2} \mathrm{O}\right)(99.9 \%$ trace metals basis), cadmium acetate dihydrate $\left(\mathrm{Cd}\left(\mathrm{CH}_{3} \mathrm{COO}\right)_{2} \cdot 2 \mathrm{H}_{2} \mathrm{O}\right) \quad(>98 \%)$, sodium myristate (>99\%), technical grade 1-octadecene (ODE), selenium (Se) (99.9\% trace metals basis), sulfur (S) (99.9\% trace metals basis), and technical grade oleic acid (OA) (90\%) were purchased from Sigma Aldrich. Hexane, ethanol, and methanol were purchased from Merck Millipore.

2.1.2. Preparation of Cadmium Myristate. Cadmium myristate was synthesized by following a previously reported protocol. ${ }^{1}$ For the preparation of cadmium myristate, $1.23 \mathrm{~g}$ of cadmium nitrate tetrahydrate was dissolved in $40 \mathrm{~mL}$ of methanol and $3.13 \mathrm{~g}$ of sodium myristate was dissolved in $250 \mathrm{~mL}$ of methanol by continuous stirring. After the complete dissolution, both solutions were mixed and stirred for around $1 \mathrm{~h}$. Subsequently, bulky solutions of cadmium myristate were centrifuged and precipitated in methanol. For the complete removal of excess precursors and better purification, this procedure was repeated three times. Lastly, the precipitated bulk was dried under vacuum overnight.

2.1.3. Synthesis of the $4 \mathrm{ML}$ CdSe Core NPLs. For the synthesis, $340 \mathrm{mg}$ of cadmium myristate, $24 \mathrm{mg}$ of Se, and $30 \mathrm{~mL}$ of ODE were loaded into a three-neck flask as given in the protocol. ${ }^{31}$ The solution 
was degassed at $95{ }^{\circ} \mathrm{C}$ for $1 \mathrm{~h}$. Then, the temperature was set to 240 ${ }^{\circ} \mathrm{C}$ under an argon atmosphere. When the temperature reached 195 ${ }^{\circ} \mathrm{C}, 120 \mathrm{mg}$ of cadmium acetate dihydrate was added to the reaction solution. After $10 \mathrm{~min}$ growth at $240{ }^{\circ} \mathrm{C}, 1 \mathrm{~mL}$ of OA was injected and the solution was cooled to room temperature using a water bath. Below $70^{\circ} \mathrm{C}, 5 \mathrm{~mL}$ of hexane was injected for better dissolution of NPLs. NPLs were precipitated by the addition of ethanol and kept in hexane solution following the previously reported procedures. ${ }^{31}$

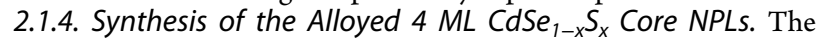
synthesis of alloyed $4 \mathrm{ML} \mathrm{CdSe} e_{1-x} S_{x}$ core NPLs was performed by following the previous literature with few modifications. ${ }^{27}$ For the synthesis of $4 \mathrm{ML} \mathrm{CdSe} e_{1-x} \mathrm{~S}_{x}$ alloyed core NPLs, $340 \mathrm{mg}$ of cadmium myristate, $16 \mathrm{mg}$ of $\mathrm{Se}$, and $30 \mathrm{~mL}$ of ODE were added into a $100 \mathrm{~mL}$ three-neck flask. The solution was degassed under vacuum at $95{ }^{\circ} \mathrm{C}$ for around $30 \mathrm{~min}$. Then, the temperature of the solution was set to $240{ }^{\circ} \mathrm{C}$ under argon gas. At $100{ }^{\circ} \mathrm{C}$, the desired amount of sulfur precursor (S/ODE, $0.15 \mathrm{M}$ ) was injected rapidly as we have optimized our experimental procedure in this way to avoid the otherwise possible formation of CdS NPLs. For the synthesis of $4 \mathrm{ML}$ $\mathrm{CdSe}_{1-x} \mathrm{~S}_{x}$ alloyed core NPLs with sulfur compositions $(x)$ of 0.65 , 0.75 , and 0.80 , the sulfur precursor of $1.0,2.0$, and $3.0 \mathrm{~mL}$ in volume were added. When the temperature reached between $\sim 180$ and 200 ${ }^{\circ} \mathrm{C}, 120 \mathrm{mg}$ of cadmium acetate dihydrate was added. The addition of different amounts of sulfur precursor results in different growth kinetics of $4 \mathrm{ML}$ magic-sized seeds. Thus, the addition of cadmium acetate powder requires different temperatures for different alloyed samples (e.g., $\sim 180-200{ }^{\circ} \mathrm{C}$ ). This temperature was decided by observing the appearance of golden yellow color for the magic-sized seeds of different samples. When we increased the $S$ concentration, the temperature for the formation of $4 \mathrm{ML} \mathrm{CdSe}_{1-x} \mathrm{~S}_{x}$ seed is lowered. This has been checked and optimized during the synthesis. After 10 min growth at $240{ }^{\circ} \mathrm{C}, 1 \mathrm{~mL}$ of $\mathrm{OA}$ was injected and the solution was cooled to room temperature using a water bath as seen in Figure 1a. Below $70{ }^{\circ} \mathrm{C}, 5 \mathrm{~mL}$ of hexane was injected for better dissolution of NPLs. For the cleaning, NPLs were precipitated by the addition of ethanol and kept in hexane solution using size-selective precipitation. To remove the extra subpopulation of NPLs emitting in the green along with blue-emitting alloyed NPLs, the ensemble NPLs were centrifuged at $2000 \mathrm{rpm}$, which allowed the extra green-emitting ones selectively to settle down.

2.1.5. Preparation of Crown Precursors. Cadmium and sulfur precursors were prepared according to the established protocol in ref 32. Four hundred eighty milligrams of cadmium acetate dihydrate, $340 \mu \mathrm{L}$ of OA, and $2 \mathrm{~mL}$ of ODE were loaded in a $50 \mathrm{~mL}$ three-neck flask. The solution was heated to $100{ }^{\circ} \mathrm{C}$ under an ambient atmosphere with stirring and sonicating. Heating and sonication were repeated until a whitish homogeneous gel was obtained. Next, this growth solution was used for the coating of CdS crown on the alloyed NPLs.

2.1.6. Synthesis of $4 M L C d S e_{1-x} S_{x} / C d S$ Core/Crown NPLs. Five milliliters of ODE, $100 \mu \mathrm{L}$ of OA, and $1 \mathrm{~mL}$ of $\mathrm{CdSe}_{1-x} \mathrm{~S}_{x}(100 \mu \mathrm{L}$ of $\mathrm{CdSe}_{1-x} \mathrm{~S}_{x}$ NPLs dissolved in $3 \mathrm{~mL}$ of hexane having an optical density of $\sim 1$ at $350 \mathrm{~nm}$ ) were loaded into a $50 \mathrm{~mL}$ three-neck flask and degassed at $90{ }^{\circ} \mathrm{C}$. Under argon flow, the temperature of the solution was increased up to $230^{\circ} \mathrm{C}$. Around $210^{\circ} \mathrm{C}$, Cd-acetate in an ODE solution was injected, followed by the injection of $0.5 \mathrm{~mL}$ of sulfur $(0.15 \mathrm{M}$ in ODE) at a rate of $4 \mathrm{~mL} / \mathrm{h}$. After the injection of sulfur, $\mathrm{CdSe}_{1-x} \mathrm{~S}_{x} / \mathrm{CdS}$ core/crown NPLs were annealed for $3 \mathrm{~min}$ at $220{ }^{\circ} \mathrm{C}$ and cooled down to room temperature using a water bath, as seen in Figure $1 \mathrm{~b}$. Ethanol was used for precipitation, and the precipitated samples were dissolved in hexane.

2.1.7. Synthesis of $\mathrm{ZnO}$ Nanoparticles. To synthesize $\mathrm{ZnO}$ nanoparticles, $3 \mathrm{mmol} \mathrm{Zn}$ acetate (anhydrous) was first dissolved in $30 \mathrm{~mL}$ of dimethyl sulfoxide solution. Then, $10 \mathrm{~mL}$ of ethanol solution dissolved with $5 \mathrm{mmol}$ tetramethylammonium hydroxide was introduced to the above $\mathrm{Zn}$ solution dropwise and the final solution was stirred for $1 \mathrm{~h}$ under ambient conditions. The $\mathrm{ZnO}$ nanoparticles were precipitated with an excessive amount of acetone solution and then completely redispersed into the ethanol solution. The solutions were filtered before use.
2.2. Fabrication and Characterization of LEDs. 2.2.1. Device Fabrication. ITO substrates were cleaned by sonication with deionized water, acetone, and isopropyl alcohol for $10 \mathrm{~min}$, respectively. Then, UV ozone treatment was performed for $10 \mathrm{~min}$. It was followed with the spin-coating of the PEDOT:PSS solution on ITO substrates at $4000 \mathrm{rpm}$ for $60 \mathrm{~s}$, and the substrates were heated at $130{ }^{\circ} \mathrm{C}$ for $20 \mathrm{~min}$. PVK $(8 \mathrm{mg} / \mathrm{mL})$ was spin-coated at $4000 \mathrm{rpm}$ for $30 \mathrm{~s}$ and heated at $120^{\circ} \mathrm{C}$ for $30 \mathrm{~min}$. After that, blue-emitting $4 \mathrm{ML}$ $\mathrm{CdSe}_{0.20} \mathrm{~S}_{0.80} / \mathrm{CdS}$ core/crown NPLs $(\sim 10 \mathrm{mg} / \mathrm{mL})$ were spin-coated at $2000 \mathrm{rpm}$ for $30 \mathrm{~s}$. The $\mathrm{ZnO}$ nanoparticles $(30 \mathrm{mg} / \mathrm{mL})$ were deposited at $2000 \mathrm{rpm}$ for $30 \mathrm{~s}$ and heated at $100{ }^{\circ} \mathrm{C}$ for $30 \mathrm{~min}$. The active area of each device was measured to be $4 \mathrm{~mm}^{2}$ from the overlap of ITO and Al.

2.2.2. Electrical Characterization. The CIE coordinates and EL spectra were taken using a PR705 Spectra Scan spectrometer. All EL spectra measurements were conducted at room temperature in the air with an encapsulated device. By utilizing a computer-controlled source meter (a programmable Agilent B2902A source meter and a Konica-Minolta LS-110 luminance meter in air at room temperature), the current density-voltage-luminance properties were measured simultaneously. The EQE values were calculated from the luminance, current density, and EL spectrum.

2.2.3. Optical Characterization. UV-vis absorption and photoluminescence spectra of NPLs were recorded using Shimadzu UV1800 spectrophotometer and Shimadzu RF-5301 PC spectrofluorophotometer. Our PL-QY measurement setup was equipped with an Ocean Optics S4000 spectrometer, and an integrating sphere was used. For the PL-QY measurements, the prepared samples dispersed in hexane solutions with an optical density of $\sim 0.2$ in $1.0 \mathrm{~cm}$ path length were employed (excited at a wavelength of $405 \mathrm{~nm}$ (Cobolt Laser)).

2.2.4. Structural and Elemental Characterization. Transmission electron microscopy (TEM) images of NPLs were performed with JEOL JEM $2100 \mathrm{~F}$ operated at $200 \mathrm{kV}$ in the high-angle annular darkfield scanning transmission electron microscopy (HAADF-STEM) configuration. For the sample preparation, NPLs were cleaned thoroughly for the excess ligands, and $5 \mu \mathrm{L}$ of diluted NPL solution was dropped on a copper grid and dried.

X-ray photoelectron spectroscopy (XPS) measurements were conducted using Thermo Scientific K-Alpha X-ray photoelectron spectrometer to study the elemental compositions of $\mathrm{CdSe}_{1-x} \mathrm{~S}_{x}$ core NPLs and their heterostructures. The samples were spin-coated on the silicon substrates. The XPS spectra were analyzed using Avantage/ Kratos software. The XPS spectra were corrected to the C 1s peak. The binding energy of the $\mathrm{C} 1$ s peak is $284.8 \mathrm{eV}$ and the spectrum is shifted to the $\mathrm{C} 1 \mathrm{~s}$ peak.

Time-resolved photoluminescence (TRF) measurements were conducted. A time-correlated single-photon counting system with time resolution down to 4 ps (PicoHarp 300) and the capability of delivering laser pulses with an $80 \mathrm{MHz}$ repetition rate was used. It includes a picosecond pulsed laser with an output photon energy of $3.31 \mathrm{eV}(375 \mathrm{~nm})$ driven by a driver module (PDL- 800 series) and a fast photomultiplier tube (Hamamatsu H5783 series) that is able to resolve the lifetimes on the order of a few picoseconds.

\section{RESULTS AND DISCUSSION}

In this work, alloyed CdSe $e_{1-x} S_{x}$ NPLs together with their core/ crown heterostructures emitting in the green to blue regions were investigated to achieve highly efficient emission for lightemitting applications. Among other CdSe NPLs, 3 ML CdSe core NPLs have blue emission (shown in Figure S1). These 3 ML core NPLs with a thickness of $\sim 0.9 \mathrm{~nm}$ feature weak blue emission, along with the emission coming from the mid-band gap trap states and possible subpopulations of NPLs with other thicknesses. ${ }^{21}$ These synthesized 3 ML NPLs possess a low QY $(\sim 5 \%)$, which is consistent with the previous study. ${ }^{21}$ Also, they are rolled up, which affects the film formation and hence the device performance. Due to these limitations, alloying of 
(a)

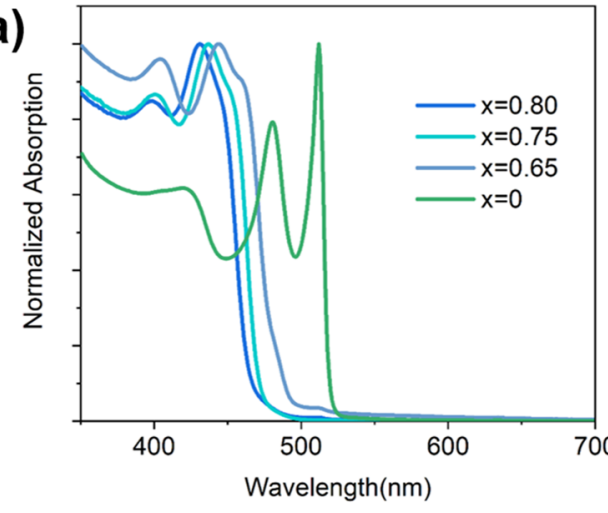

(c)

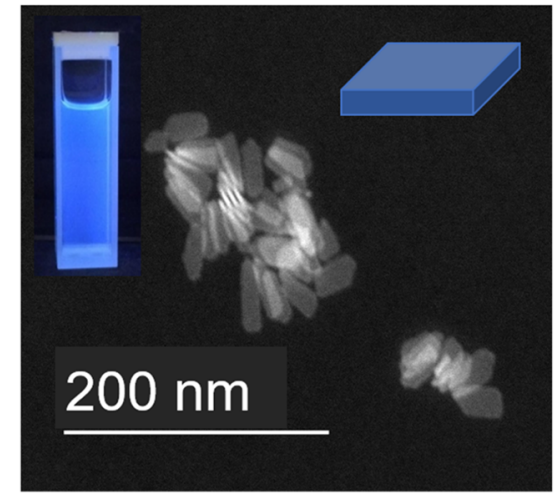

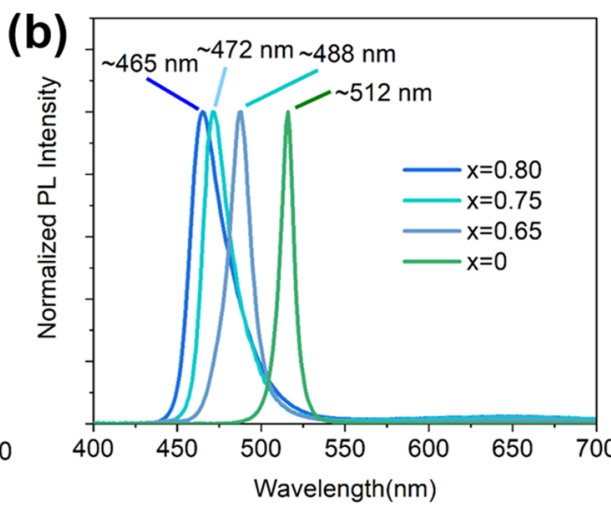

(d)

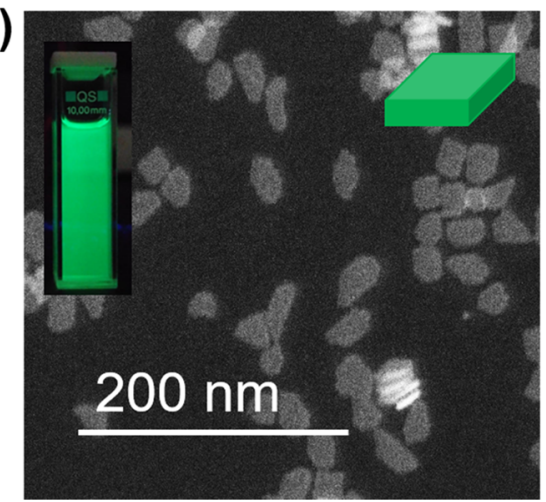

Figure 2. (a, b) Normalized absorption and photoluminescence spectra of our alloyed 4 ML CdSe $e_{1-x} \mathrm{~S}_{x}$ core NPLs with $x=0.00, x=0.65, x=0.75$, and $x=0.80$. (c) HAADF-STEM image of alloyed $4 \mathrm{ML} \mathrm{CdSe}_{1-x} S_{x}$ NPLs for the case of $x=0.80$. (d) HAADF-STEM image of the control group of $4 \mathrm{ML}$ CdSe NPLs. Insets of (c) and (d) show the digital color images of the corresponding samples excited with UV lamp, along with a schematic of a single NPL.

sulfur in green-emitting $4 \mathrm{ML}$ thick CdSe NPLs is envisioned to tune the emission spectra to the blue region. The absorption and photoluminescence spectra of $4 \mathrm{ML}$ CdSe core NPLs are presented in Figure 2. These show a narrow emission bandwidth (FWHM $\sim 10 \mathrm{~nm}$ ) with a PL-QY of $\sim 30 \%$. The absorption spectra also indicate the corresponding sharp excitonic features of e-lh $\sim 480 \mathrm{~nm}$ and e-hh $\sim 512 \mathrm{~nm}$ absorption transitions. The PL emission peaks are at $512 \mathrm{~nm}$. Also, 4 ML CdSe core NPLs have lateral dimensions of $16.2 \pm$ $2.3 \mathrm{~nm}$ by the width of $12.5 \pm 1.8 \mathrm{~nm}$. These NPLs have reduced lateral extension, leading to fewer defects and higher PL-QY. Therefore, they are more stable colloidally to form a uniform film.

These $4 \mathrm{ML}$ thick CdSe core NPLs were used as a starting material for studying the effects of sulfur alloying. Using sulfur precursor to alloy $4 \mathrm{ML}$ CdSe NPLs, $4 \mathrm{ML} \mathrm{CdSe}{ }_{1-x} \mathrm{~S}_{x} \mathrm{NPLs}$ were synthesized with the injection of different amounts of sulfur precursor solution (such as $x=0.65,0.75$, and 0.80 ). The amount of injected precursor tunes the $\mathrm{CdSe}_{1-x} \mathrm{~S}_{x}$ composition.

The formation of alloyed $\mathrm{CdSe}_{1-x} \mathrm{~S}_{x}$ leads to a blue-shift of absorption and PL spectra because of the larger band gap energy of $\mathrm{CdSe}_{1-x} \mathrm{~S}_{x}$ compared to that of pure CdSe. This has been similarly shown for CdSe quantum dots alloyed with S. ${ }^{33,34}$

As seen in Figure $2 b$, the PL emission peak wavelengths obtained for $\mathrm{CdSe}_{1-x} \mathrm{~S}_{x}$ NPLs were observed at $\sim 465, \sim 472$, and $\sim 488 \mathrm{~nm}$ for different amounts of sulfur precursor $(x=$ $0.80, x=0.75$, and $x=0.65)$ used in the synthesis process. However, we did not observe a further shift in the emission wavelength below $460 \mathrm{~nm}$ with the increased addition of sulfur precursor (Figure S2). Figure 2 shows the absorption and photoluminescence spectra of NPLs synthesized using different amounts of sulfur precursor $(x=0.00, x=0.65, x=0.75$, and $x$ $=0.80)$. In the case of alloyed NPLs, e-lh and e-hh peaks are blue shifted as compared to those of the control group of $4 \mathrm{ML}$ CdSe NPLs appearing at $\sim 475,452$, and $445 \mathrm{~nm}$ for e-lh, and $\sim 450,437$, and $432 \mathrm{~nm}$ for e-hh, respectively, for $x=0.65, x=$ 0.75 , and $x=0.80$. The FWHM of 15,17 , and $24 \mathrm{~nm}$ were observed for the corresponding samples emitting at $\sim 488,472$, and $465 \mathrm{~nm}$, respectively. The maximum PL-QY of $\sim 10 \%$ was observed for these alloyed samples shown in Figure $2 b$. Increasing sulfur precursor concentrations beyond $x=0.80$, the $\mathrm{PL}$ emission spectra show that there were two different subpopulations of NPLs along with an excess amount of colloidal quantum dots, which could not be separated from each other using the size-selective precipitation method (Figure S2). Thus, in our experiments, we could not shift the emission spectrum of alloyed $4 \mathrm{ML} \mathrm{CdSe} e_{1-x} \mathrm{~S}_{x}$ NPLs below $460 \mathrm{~nm}$. The high-angle annular dark-field scanning transmission electron microscopy (HAADF-STEM) images of alloyed $4 \mathrm{ML} \mathrm{CdSe}{ }_{1-x} \mathrm{~S}_{x}$ core NPLs with $x=0.80$ show a rectangular lateral shape with average dimensions of $29.8 \pm 1.7$ $\mathrm{nm}$ by $13.6 \pm 1.1 \mathrm{~nm}$. As the amount of sulfur is increased, the rectangular shape of their alloyed core NPLs is retained (Figure S3).

The absorption and photoluminescence spectra of alloyed 4 $\mathrm{ML} \mathrm{CdSe}_{1-x} \mathrm{~S}_{x}$ NPLs at the emission wavelength $\sim 465 \pm 1 \mathrm{~nm}$ obtained at different injection temperatures of $\mathrm{Cd}$-acetate are given in Figure S4. The emission wavelength peak of $\sim 465 \mathrm{~nm}$ with an FWHM of $\sim 24 \mathrm{~nm}$ indicates a relatively pure population of NPLs after the injection of Cd-acetate powder at 
(a)
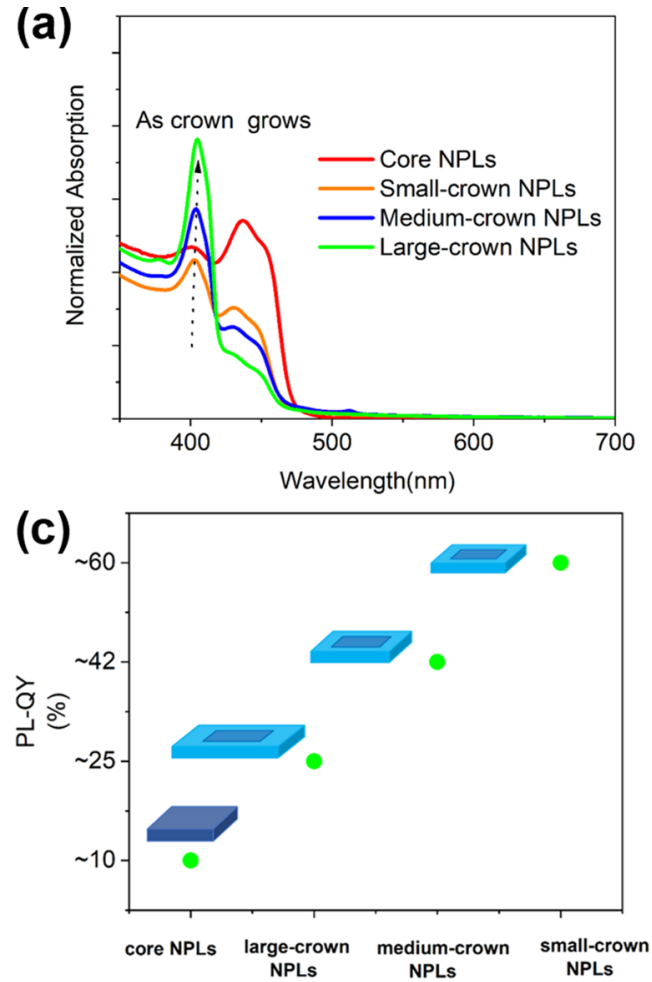

(b)

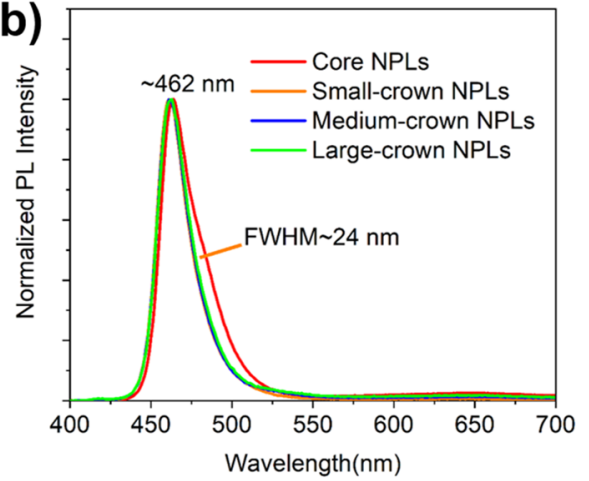

(d)

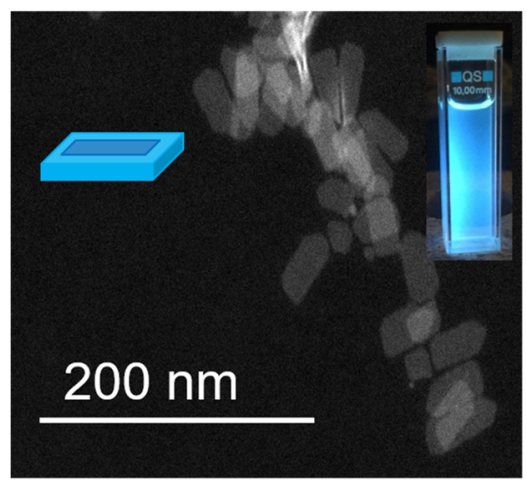

Figure 3. (a, b) Normalized absorption and photoluminescence spectra of our alloyed $4 \mathrm{ML} C \mathrm{CSe} \mathrm{e}_{1-x} \mathrm{~S}_{x} / \mathrm{CdS}$ core/crown NPLs at an emission peak of $\sim 462 \mathrm{~nm}$ having different CdS crown sizes. (c) PL-QY results of these alloyed $4 \mathrm{ML} \mathrm{CdSe} \mathrm{C}_{0.20} \mathrm{~S}_{0.80}$ core/crown NPLs with the emission peak of $\sim 462 \mathrm{~nm}$ having different CdS crown sizes. (d) HAADF-STEM images of alloyed $4 \mathrm{ML}$ alloyed CdSe $e_{0.20} \mathrm{~S}_{0.80} / \mathrm{CdS}$ core/crown NPLs. Inset shows the blue-emitting core-crown NPLs under UV lamp, along with the sketch of a single NPL.

$196{ }^{\circ} \mathrm{C}$. The temperature of cadmium precursor injection, the growth time, and the sulfur reactivity are found to be important parameters for obtaining a pure population of alloyed $4 \mathrm{ML} \mathrm{CdSe} e_{1-x} S_{x}$ NPLs. The lateral sizes of the synthesized NPLs depend on the amounts of precursors and the duration for which the reaction was maintained.

The maximum PL-QY observed for the blue-emitting alloyed core NPLs is found to be around $10 \%$. Since the dimensions of our alloyed 4 ML NPLs are relatively smaller than those of $3 \mathrm{ML}$ CdSe NPLs (which results in the alloyed NPLs, being larger and thinner, rolling up), it is possible to grow $\mathrm{CdS}$ crown around the periphery of these alloyed NPLs. Using alloyed $\mathrm{CdSe}_{1-x} \mathrm{~S}_{x}$ NPLs as the seed material, $\mathrm{CdSe}_{1-x} \mathrm{~S}_{x} / \mathrm{CdS}$ core/crown NPLs were synthesized with a slow injection of crown precursors at a higher temperature of $\sim 210^{\circ} \mathrm{C}$ by following an earlier crown synthesis recipe. ${ }^{31}$ The crown growth reduces the amount of defected NPLs with surface trap states. The addition of a CdS crown passivates the periphery of these alloyed core NPLs and results in an increase in their PL-QY as reported earlier. ${ }^{35}$

The absorption and photoluminescence spectra of the alloyed $4 \mathrm{ML} \mathrm{CdSe}{ }_{1-x} \mathrm{~S}_{x} / \mathrm{CdS}$ core/crown NPLs at a PL peak of $\sim 462 \mathrm{~nm}$ having different CdS crown sizes are shown in Figure 3b. Different crown sizes (small, medium, and large) are obtained by altering the amount of injected crown-growth precursors. The excitonic emission peak of alloyed $4 \mathrm{ML}$ $\mathrm{CdSe}_{1-x} \mathrm{~S}_{x} / \mathrm{CdS}$ core/crown NPLs is still in the same spectral position for even different crown widths due to the same vertical thickness of the crown and core regions. As shown in Figure $3 \mathrm{a}$, the absorption spectrum has an additional peak at $\sim 405 \mathrm{~nm}$, which is similarly reported for CdS crown deposited around $4 \mathrm{ML}$ thick core NPLs. Furthermore, $\mathrm{CdSe}_{1-x} \mathrm{~S}_{x} / \mathrm{CdS}$ core/crown NPLs demonstrate a small blue-shift $(\sim 3 \mathrm{~nm})$ in the emission spectrum with respect to that of the core NPLs. This could be the result of the additional alloying of $\mathrm{CdSe}_{1-x} \mathrm{~S}_{x}$ core NPLs during the CdS crown deposition. Small-crown deposition around alloyed NPLs is observed to have a considerable increase in the PL-QYs (from 10 to $60 \%$ ). However, the PL-QYs of the medium and large-crown grown NPLs could only reach $\sim 42$ and $\sim 25 \%$, respectively. The increased lateral size of the $\mathrm{CdS}$ crown increases the trap site density due to the larger surface area, which diminishes the increase in the resulting PL-QY. HAADF-STEM images of 4 ML alloyed $\mathrm{CdSe}_{1-x} \mathrm{~S}_{x} / \mathrm{CdS}$ core/crown NPLs with $x=0.80$ are shown in Figure 3. As shown in Figure 3b, as the crown is grown on $4 \mathrm{ML}$ alloyed $\mathrm{CdSe}_{1-x} \mathrm{~S}_{x}$ core NPLs, there is a decrease in FWHM.

Also, the photoluminescence emission spectra of alloyed 4 ML CdSe ${ }_{1-x} \mathrm{~S}_{x} / \mathrm{CdS}$ core/crown NPLs with the PL emission peaks of $\sim 462,471$, and $487 \mathrm{~nm}$ using $x=0.80, x=0.75$, and $x$ $=0.65$ are shown in Figure S5.

Lateral sizes of the $4 \mathrm{ML}$ alloyed $\mathrm{CdSe}_{1-x} \mathrm{~S}_{x} / \mathrm{CdS}$ core/ crown NPLs are increased compared to those of $4 \mathrm{ML}$ alloyed $\mathrm{CdSe}_{1-x} \mathrm{~S}_{x}$ core NPLs while their thicknesses remain the same. These NPLs have a rectangular shape with a length of $34.0 \pm$ $4.3 \mathrm{~nm}$ and a width of $14.7 \pm 2.1 \mathrm{~nm}$. Also, $4 \mathrm{ML}$ alloyed $\mathrm{CdSe}_{1-x} \mathrm{~S}_{x} / \mathrm{CdS}$ core/medium-crown sized NPLs have the length of $35.0 \pm 2.5 \mathrm{~nm}$ and the width of $15.2 \pm 1.4 \mathrm{~nm}$, while $4 \mathrm{ML}$ alloyed $\mathrm{CdSe}_{1-x} \mathrm{~S}_{x} / \mathrm{CdS}$ core/large-crown sized NPLs possess the lateral dimensions of length of $36.1 \pm 3.2 \mathrm{~nm}$ and width of $16.5 \pm 2.5 \mathrm{~nm}$. HR-TEM images of alloyed $4 \mathrm{ML}$ $\mathrm{CdSe}_{1-x} \mathrm{~S}_{x}$ NPLs core and core-crown with various precursor ratios $x=0.65$ and $x=0.75$ are also shown in Figure S3. They 
possess a rectangular shape regardless of the sulfur composition.

To investigate the elemental composition of samples, X-ray photoelectron spectroscopy (XPS) is performed. All recorded XPS spectra have been shifted to standard C 1s spectra positioned at $284.8 \mathrm{eV}$. High-resolution XPS spectra of Cd 3d and $S 2 p$ peaks for the core NPL and core-crown NPLs are shown (Figure S6). In the high-resolution XPS spectra, $S$ peaks observed for the alloyed $4 \mathrm{ML} C \mathrm{dSe}_{0.20} \mathrm{~S}_{0.80}$ core NPLs can be fitted with four peaks, $S 2 p_{1 / 2}$ and $S 2 p_{3 / 2}$ (positioned at 162.3 and $161.1 \mathrm{eV}$ ) and $\mathrm{Se} 3 \mathrm{p}_{3 / 2}$ and $\mathrm{Se} 3 \mathrm{p}_{1 / 2}$ (positioned at 165.7 and $159.6 \mathrm{eV}$ ) (Figure S6). On the contrary, we only see $\mathrm{S}$ $2 \mathrm{p}_{1 / 2}$ and $\mathrm{S} 2 \mathrm{p}_{3 / 2}$ (positioned at 162.8 and $161.7 \mathrm{eV}$ ) in the XPS spectra of alloyed $4 \mathrm{ML} \mathrm{CdSe} e_{0.20} \mathrm{~S}_{0.80} / \mathrm{CdS}$ core/crown NPLs. Absence of Se peaks in the alloyed $4 \mathrm{ML} \mathrm{CdSe} \mathrm{e}_{0.20} \mathrm{~S}_{0.80} /$ $\mathrm{CdS}$ core/crown NPLs confirms the formation of the crown in alloyed $4 \mathrm{ML} \mathrm{CdSe} e_{0.20} \mathrm{~S}_{0.80}$ core NPLs. As the alloying value is changed between $x=0.65$ and $x=0.75$, the atomic ratio of $S$ to Se is correspondingly increased in the XPS data (Figure S7).

Furthermore, XRD measurements are performed to determine the crystal structure of alloyed $4 \mathrm{ML} \mathrm{CdSe}{ }_{1-x} \mathrm{~S}_{x}$ core and $\mathrm{CdSe}_{1-x} \mathrm{~S}_{x} / \mathrm{CdS}$ core/crown NPLs shown in Figure S8. CdSe core NPLs show a zinc-blende crystal structure with broader diffraction peaks owing to their nanocrystal size. The characteristic diffraction peaks are observed for (111), (220), and (311) planes on CdSe core NPLs (Table S1 and Figure S9). With the formation of the CdS crown region, the diffraction peaks were slightly shifted to higher angles toward CdS NPLs, verifying the formation of a crown. XRD analysis confirms that both $\mathrm{CdSe}$ and $\mathrm{CdS}$ phases have zinc-blende structures. $^{36}$

In addition, the elemental analysis with energy-dispersive $\mathrm{X}$ ray (EDX) spectroscopy is performed. With crown deposition, the concentration of sulfur increases, confirming the formation of core/crown heterostructures (Table 1).

Table 1. EDX Spectra of Our Alloyed $4 \mathrm{ML} \mathrm{CdSe} \mathrm{C}_{0.20} \mathrm{~S}_{0.80}$ Core NPLs with and $4 \mathrm{ML} \mathrm{CdSe} \mathrm{e}_{0.20} \mathrm{~S}_{0.80} / \mathrm{CdS}$ Crown NPLs with Different Sizes

\begin{tabular}{lccc}
\multicolumn{4}{c}{ chemical compositions (at. \%) } \\
\hline & $\mathrm{Cd}(\%)$ & $\mathrm{Se}(\%)$ & $\mathrm{S}(\%)$ \\
$\mathrm{CdSe}_{0.20} \mathrm{~S}_{0.80}$ core NPLs & 54 & 15 & 31 \\
$\mathrm{CdSe}_{0.20} \mathrm{~S}_{0.80} /$ CdS small-crown NPLs & 51 & 17 & 32 \\
$\mathrm{CdSe}_{0.20} \mathrm{~S}_{0.80} /$ CdS medium-crown NPLs & 47 & 18 & 35 \\
$\mathrm{CdSe}_{0.20} \mathrm{~S}_{0.80} /$ CdS large-crown NPLs & 42 & 18 & 40 \\
\hline
\end{tabular}

To understand the decay kinetics of alloyed $4 \mathrm{ML}$ $\mathrm{CdSe}_{0.20} \mathrm{~S}_{0.80}$ core and $4 \mathrm{ML} \mathrm{CdSe}{ }_{0.20} \mathrm{~S}_{0.80} / \mathrm{CdS}$ small-crown NPLs, we also performed time-resolved fluorescence spectroscopy (TRF). Fluorescence decay curves of the samples are fitted using three-exponential decay functions (see Table S2 and Figure S10). The amplitude-averaged fluorescence lifetime of alloyed $4 \mathrm{MLCdSe} \mathrm{C}_{0.20} \mathrm{~S}_{0.80}$ core NPLs and alloyed $4 \mathrm{ML}$ $\mathrm{CdSe}_{0.20} \mathrm{~S}_{0.80} / \mathrm{CdS}$ small-crown NPLs is measured to be $\sim 2.3 \pm$ 0.2 and $2.2 \pm 0.2 \mathrm{~ns}$, respectively. There is no significant reduction in a lifetime. Any small changes can be attributed to the different dielectric constants of core and core-crown structures.

$\mathrm{CdSe}_{x} \mathrm{~S}_{1-x}$-based NPLs possessing PL-QY $(\sim 10-20 \%)$ are already shown in the emission range of $\sim 490-510 \mathrm{~nm}$ and also $\mathrm{CdSe}_{x} \mathrm{~S}_{1-x} / \mathrm{CdS}$ core/crown NPLs with PL-QY of $\sim 40-50 \%$ in 500-540 nm. ${ }^{13,27}$ Also, recently Di Giacomo et al. reported that blue-emitting NPLs with photoluminescence quantum efficiencies of $\sim 30 \%$ in the emission wavelength of $459-463$ $\mathrm{nm}$ were synthesized using a modified synthesis protocol. ${ }^{37}$ In comparison to previous work, we synthesized highly efficient with $\mathrm{CdSe}_{1-x} \mathrm{~S}_{x} / \mathrm{CdS}$ core/crown NPLs in the spectral range of $\sim 462-487 \mathrm{~nm}$ with PL-QY of $\sim 60 \%$. These results are highly promising for light-emitting diode applications.

To evaluate the electroluminescence (EL) properties of devices based on the blue-emitting $4 \mathrm{ML} \mathrm{CdSe}{ }_{0.20} \mathrm{~S}_{0.80} / \mathrm{CdS}$ core/small-crown NPLs, NPL-LEDs with the conventional device architecture of indium tin oxide (ITO)/PEDOT:PSS/ $\mathrm{PVK} / \mathrm{NPLs} /$ zinc oxide $(\mathrm{ZnO}) / \mathrm{NPLs} / \mathrm{Al}$ are fabricated as shown in Figure 4a. In this conventional LED architecture, these layers consist of ITO as the anode, a $\mathrm{ZnO}$ nanocrystal film $(30 \mathrm{~nm})$ as an electron-injecting layer, the emissive layer of blue-emitting $4 \mathrm{ML} \mathrm{CdSe}{ }_{0.20} \mathrm{~S}_{0.80} / \mathrm{CdS}$ core/small-crown NPLs $(\sim 40 \mathrm{~nm})$, PEDOT:PSS $(\sim 50 \mathrm{~nm})$, and PVK layer $(\sim 40 \mathrm{~nm})$ as a hole-transporting layer, and $\mathrm{Al}(\sim 100 \mathrm{~nm})$ as

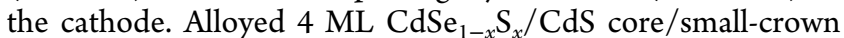
NPLs with $x=0.80$ are selected as an emitter because their PL emission wavelengths lie in the blue region $(\sim 468 \mathrm{~nm})$. To select a suitable HTL layer with our NPLs, ultraviolet photoelectron spectroscopy (UPS) measurement is carried out on blue-emitting alloyed $4 \mathrm{ML} \mathrm{CdSe} e_{0.20} \mathrm{~S}_{0.80} / \mathrm{CdS}$ core/ small-crown NPLs (Figures $4 \mathrm{~b}$ and S11). The conduction and valence bands are measured to be 3.6 and $6.2 \mathrm{eV}$, respectively (Figure S12). Due to this, PVK is chosen as the HTL material to lower the hole injection barrier between the holetransporting and electron-emitting layer interfaces for blueemitting NPL-LEDs similar to that previously reported for blue-emitting QD-LEDs. ${ }^{38}$ PVK is well aligned with the valence band of emitting nanoplatelets as seen in Figure $4 \mathrm{~b}$. The hole-transporting layer PVK possesses hole mobility of $\sim 10^{-7}-10^{-6} \mathrm{~cm}^{2} /\left(\begin{array}{ll}\mathrm{V} & \mathrm{s}\end{array}\right){ }^{7}$ As shown in Figure $4 \mathrm{~b}, \mathrm{ZnO}$ nanoparticles with conduction and valence bands of $\sim 4.0$ and $\sim 7.4 \mathrm{eV}$ appear to be ideal ETL to increase the radiative recombination at the emitting region. In addition, $\mathrm{ZnO}$ nanoparticles are known as an efficient electron injection layer due to their high electron mobilities $\left(\sim 1.8 \times 10^{-3} \mathrm{~cm}^{2} /\right.$ $(\mathrm{V} \mathrm{s})) .^{39}$

The roughness of each layer is measured using atomic force microscopy (AFM). These measurements confirm surface roughness ( $\mathrm{Rq}$ ) of less than $5 \mathrm{~nm}$ (Figure S13). In previous reports, NPL films are demonstrated to have a similar level of surface roughness of $\sim 4 \mathrm{~nm}$. $^{40}$ Thus, these smooth surfaces may prevent failures in devices during operation due to the development of short-circuiting current pathways possibly arising from the rough spots across the device.

The current density-luminance-voltage characteristics of the devices are shown in Figure 5a. As seen, the current density and luminance increase with the applied voltage. The turn-on voltage of $\sim 4 \mathrm{~V}$, a maximum brightness of $\sim 12 \mathrm{~cd} / \mathrm{m}^{2}$ under 5 $\mathrm{V}$, and EQE of $\sim 0.06 \%$ are achieved. There is no parasitic emission from any of the other layers in our measurement. However, the EL spectra of these NPL-LEDs exhibit a red-shift and broadening compared to PL as shown in Figure 5c,d. These can be associated with the optical phonon coupling and quantum-confined Stark effect, which is Joule heating during operation. $^{41,42}$ Furthermore, EL emission is shown in Figure 5c. The CIE 1931 coordinates of blue-emitting $4 \mathrm{ML}$ $\mathrm{CdSe}_{0.20} \mathrm{~S}_{0.80} / \mathrm{CdS}$ core/small-crown NPLs are $(0.23,0.14)$. The image given in the inset shows blue emission from an NPL-LED (Figure 5c). 
(a)
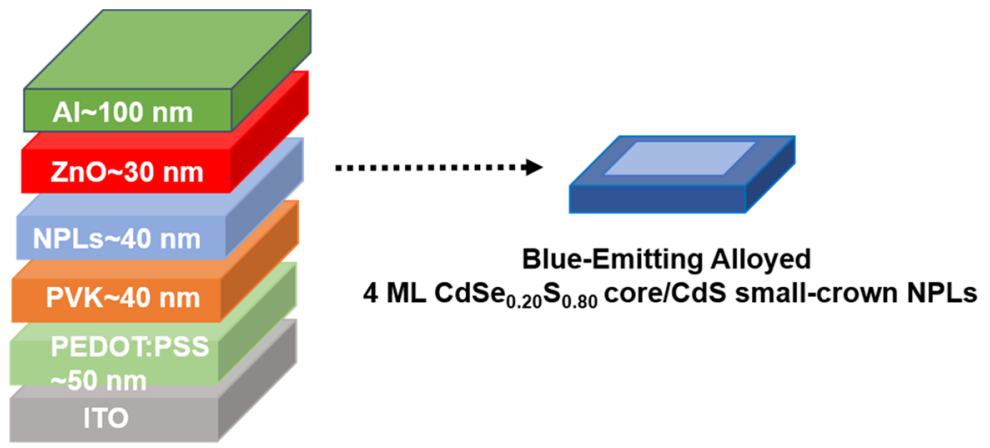

(b)

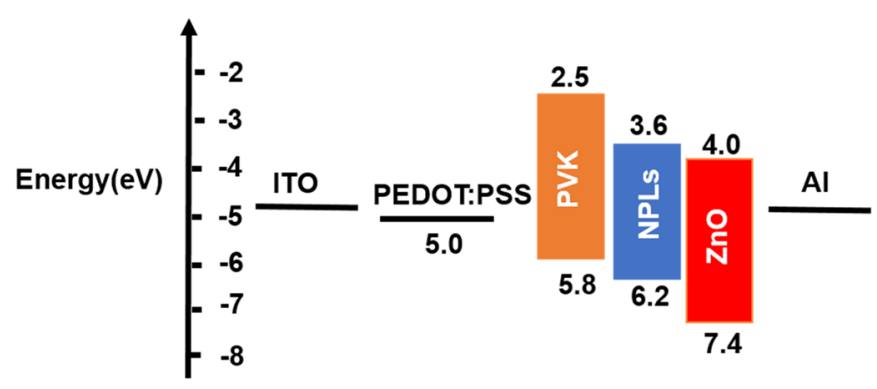

Figure 4. (a) Schematic representation of NPL-LED architecture and (b) schematic band energy diagram of NPL-LEDs. The values of 4 ML $\mathrm{CdSe}_{0.20} \mathrm{~S}_{0.80} / \mathrm{CdS}$ core/small-crown NPLs were obtained by ultraviolet photoelectron spectroscopy (UPS).

(a)

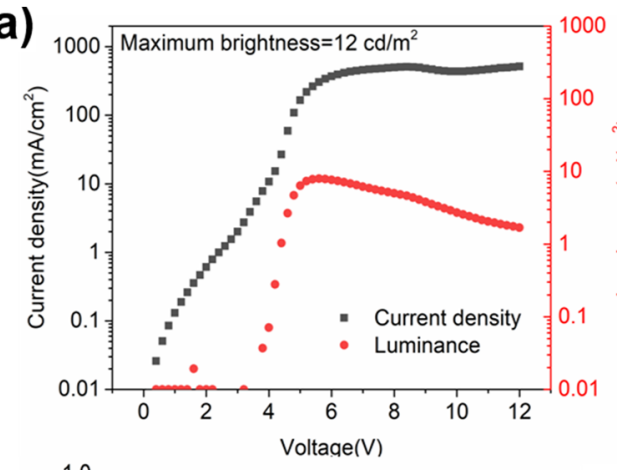

(b)

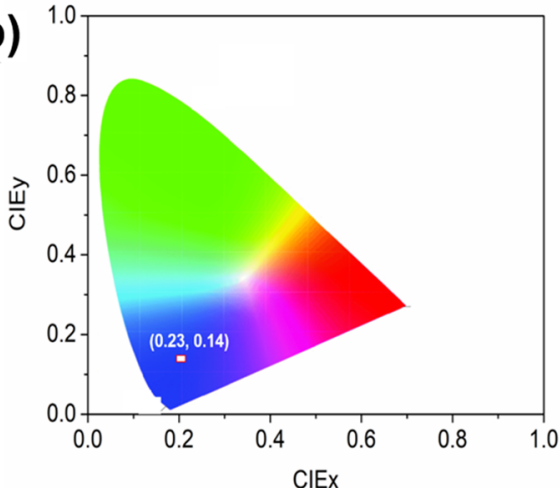

(c)

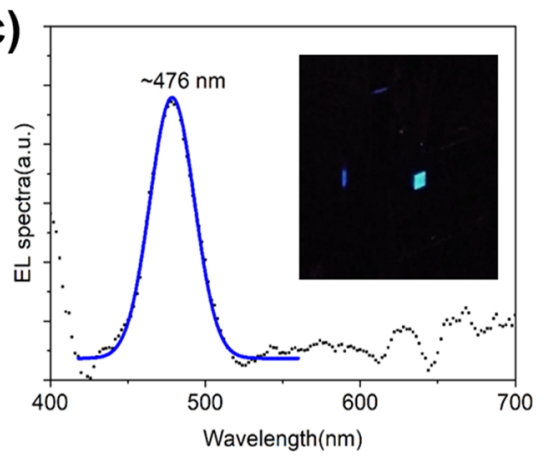

(d)

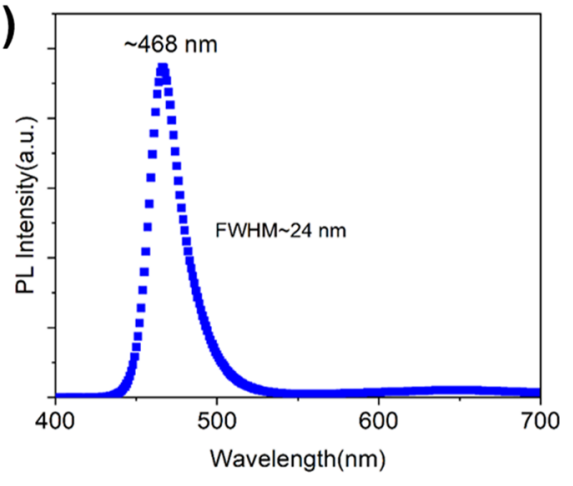

Figure 5. (a) Current density-luminance-voltage characteristics of NPL-LEDs. (b) Position of the coordinates of (0.23, 0.14) in the CIE diagram. (c) Electroluminescence spectra of NPL-LEDs with the inset showing an image of the fabricated LEDs. (d) Photoluminescence spectra of 4 ML $\mathrm{CdSe}_{0.20} \mathrm{~S}_{0.80} / \mathrm{CdS}$ core/crown NPLs.

There are few reports in the literature about blue QD-LED but no report about NPL-based on blue LED. Ji et al. reported the devices from core/shell CdSe/ZnS QDs with EL emission of $478 \mathrm{~nm}$ and demonstrated a low turn-on voltage of $2.8 \mathrm{~V}$ and a maximum brightness of $18127 \mathrm{~cd} / \mathrm{m}^{2}$. ${ }^{44}$ Also, Shen et al. exhibited blue quantum dot LEDs based on $\mathrm{CdSe} / \mathrm{ZnSe}$ core/ shell structures with a maximum brightness of $62600 \mathrm{~cd} / \mathrm{m}^{2}$, a low turn-on voltage of $2.5 \mathrm{~V}$, and EL spectra of $476 \mathrm{~nm} .{ }^{43}$ In the previous reports of blue-emitting QD-LED, the authors used core/shell $\mathrm{CdSe} / \mathrm{ZnS}$ or $\mathrm{ZnSe} \mathrm{QD}$ heterostructures to emit in the blue region and these provided better stability owing to the passivation of surface states. However, in our report, we produced alloyed $\mathrm{CdSe}_{1-x} \mathrm{~S}_{x} / \mathrm{CdS}$ core-crown NPL heterostructures to obtain blue emission. With the formation core-crown heterostructures, we passivated lateral dimensions here. On the other hand, the core-shell heterostructures 
passivate surface atoms with a vertically grown shell, resulting in a red-shift due to the change in the NPL thickness; thus, the blue emission is not obtained.

Furthermore, the poor performance of blue-emitting NPLLEDs can be ascribed to the following reasons. NPLs tend to form stacks in a film, which increases the chances of exciton quenching via fast energy transfer to trap sites in the defected NPLs and thus decreases the PL-QY of NPL films, leading to lower efficiency of NPL-LEDs. Moreover, excessive ligands can form insulating layers, which may further hinder the charge injection in NPL-LEDs, although excessive ligands can passivate the surface defects and increase the PL-QY of NPLs. Hence, the control of ligand density affects the device's performance.

Moreover, blue emission creates difficulty also for the charge injection because of the large band gap energies and insufficient charge transport materials. That is why the efficiency of the blue-emitting LEDs is currently lower in general. However, these NPL-based LEDs are highly promising because of the high color purity as well as their high PL-QY and should be further explored.

\section{CONCLUSIONS}

In summary, alloyed $4 \mathrm{ML} \mathrm{CdSe} e_{1-x} \mathrm{~S}_{x}$ core NPLs together with $\mathrm{CdSe}_{1-x} \mathrm{~S}_{x} / \mathrm{CdS}$ core/crown NPLs were examined in terms of excitonic, structural, and chemical properties. Blue emission with the peak wavelength of $\sim 462-487 \mathrm{~nm}$ and PL-QY of $\sim 60 \%$ was successfully achieved with alloyed $\mathrm{CdSe}_{1-x} \mathrm{~S}_{x} / \mathrm{CdS}$ core-crown NPLs. Also, blue-emitting NPL-LEDs have been fabricated using a conventional device architecture, where these NPLs were used as emitting layers between a hole injection layer (PVK) and an electron injection layer $(\mathrm{ZnO})$. Our NPL-LEDs exhibit an FWHM of $34 \mathrm{~nm}$ and a low turn-on voltage of $\sim 4 \mathrm{~V}$, along with luminance up to $\sim 12 \mathrm{~cd} / \mathrm{m}^{2}$ and EQE of $\sim 0.06 \%$. These blue NPL-based LEDs are of great interest to achieve pure colored LEDs using NPL-based red, green, and blue LEDs. All these results indicate that these alloy-based materials are promising for display and lighting applications.

\section{ASSOCIATED CONTENT}

\section{SI Supporting Information}

The Supporting Information is available free of charge at https://pubs.acs.org/doi/10.1021/acsanm.1c03939.

(a) Normalized absorption and photoluminescence spectra of synthesized $3 \mathrm{ML}$ CdSe NPLs and (b) HAADF-TEM images of $3 \mathrm{ML}$ CdSe NPLs. (a) Normalized absorption and photoluminescence spectra and (b) HR-TEM images of alloyed $4 \mathrm{ML} \mathrm{CdSe}{ }_{1-x} S_{x}$ NPLs for the case of $x=0.85$. Normalized photoluminescence spectra of our alloyed $4 \mathrm{ML} \mathrm{CdSe} \mathrm{M}_{1-x} \mathrm{~S}_{x} /$ CdS core/small-crown NPLs. XRD spectra of our alloyed $4 \mathrm{ML} \quad \mathrm{CdSe}_{0.20} \mathrm{~S}_{0.80}$ core and $4 \mathrm{ML}$ $\mathrm{CdSe}_{0.20} \mathrm{~S}_{0.80} / \mathrm{CdS}$ core/small-crown NPLs. X-ray analysis of alloyed $4 \mathrm{ML} \mathrm{CdSe} \mathrm{C}_{0.20} \mathrm{~S}_{0.80}$ NPLs core and alloyed $4 \mathrm{ML} \mathrm{CdSe}_{0.20} \mathrm{~S}_{0.80} / \mathrm{CdS}$ core-crown NPLs. XRD spectra of our alloyed $4 \mathrm{ML} \mathrm{CdSe} e_{1-x} S_{x}$ core NPLs for $x=0.65$ and $x=0.75$. TRF decay components for our alloyed $4 \mathrm{ML} \mathrm{CdSe}{ }_{0.20} \mathrm{~S}_{0.80}$ NPLs core and alloyed $4 \mathrm{ML}$ $\mathrm{CdSe}_{0.20} \mathrm{~S}_{0.80} / \mathrm{CdS}$ core/small-crown NPLs. Atomic force microscopy images of the spin-coated (a) PEDOT:PSS and (b) PEDOT:PSS/PVK, (c) PEDOT:PSS/PVK/ blue-emitting $4 \mathrm{ML} \mathrm{CdSe} e_{0.20} \mathrm{~S}_{0.80} / \mathrm{CdS}$ core/small-crown NPLs, and (d) PEDOT:PSS/PVK/blue-emitting $4 \mathrm{ML}$ $\mathrm{CdSe}_{0.20} \mathrm{~S}_{0.80} / \mathrm{CdS}$ core/small-crown NPLs/ZnO films (PDF)

\section{AUTHOR INFORMATION}

\section{Corresponding Author}

Hilmi Volkan Demir - School of Materials Science and Engineering, Nanyang Technological University, Singapore 639798; LUMINOUS! Centre of Excellence for Semiconductor Lighting and Displays, The Photonics Institute, School of Electrical and Electronic Engineering and School of Physical and Mathematical Sciences, Nanyang Technological University, Singapore 639798; Department of Electrical and Electronics Engineering, Department of Physics, UNAM-Institute of Materials Science and Nanotechnology, Bilkent University, Ankara 06800, Turkey; (이이.org/ 0000-0003-1793-112X; Email: hvdemir@ntu.edu.sg

\section{Authors}

Merve Izmir - School of Materials Science and Engineering, Nanyang Technological University, Singapore 639798; (1) orcid.org/0000-0001-8602-0106

Ashma Sharma - LUMINOUS! Centre of Excellence for Semiconductor Lighting and Displays, The Photonics Institute, School of Electrical and Electronic Engineering, Nanyang Technological University, Singapore 639798

Sushant Shendre - LUMINOUS! Centre of Excellence for Semiconductor Lighting and Displays, The Photonics Institute, School of Electrical and Electronic Engineering, Nanyang Technological University, Singapore 639798; (1) orcid.org/0000-0001-8586-7145

Emek Goksu Durmusoglu - School of Physical and Mathematical Sciences, Nanyang Technological University, Singapore 639798; 0 orcid.org/0000-0001-6840-8342

Vijay Kumar Sharma - LUMINOUS! Centre of Excellence for Semiconductor Lighting and Displays, The Photonics Institute, School of Electrical and Electronic Engineering, Nanyang Technological University, Singapore 639798; (1) orcid.org/0000-0002-2028-5715

Farzan Shabani - Department of Electrical and Electronics Engineering, Department of Physics, UNAM-Institute of Materials Science and Nanotechnology, Bilkent University, Ankara 06800, Turkey

Hamed Dehghanpour Baruj - Department of Electrical and Electronics Engineering, Department of Physics, UNAMInstitute of Materials Science and Nanotechnology, Bilkent University, Ankara 06800, Turkey

Savas Delikanli - LUMINOUS! Centre of Excellence for Semiconductor Lighting and Displays, The Photonics Institute, School of Electrical and Electronic Engineering and School of Physical and Mathematical Sciences, Nanyang Technological University, Singapore 639798; Department of Electrical and Electronics Engineering, Department of Physics, UNAM-Institute of Materials Science and Nanotechnology, Bilkent University, Ankara 06800, Turkey; (1) orcid.org/ 0000-0002-0613-8014

Manoj Sharma - LUMINOUS! Centre of Excellence for Semiconductor Lighting and Displays, The Photonics Institute, School of Electrical and Electronic Engineering, Nanyang Technological University, Singapore 639798; (1) orcid.org/0000-0001-5215-9740 
Complete contact information is available at:

https://pubs.acs.org/10.1021/acsanm.1c03939

\section{Notes}

All the authors have read the manuscript and approved the submission.

The authors declare no competing financial interest.

\section{ACKNOWLEDGMENTS}

This research is supported by the National Research Foundation, Prime Minister's Office, Singapore under its NRF Investigatorship Award program (NRF-NRFI2016-08) and the Ministry of Education Tier 1 grant (MOE-RG62/20). H.V.D. also gratefully acknowledges the support from TUBA.

\section{REFERENCES}

(1) Tessier, M. D.; Javaux, C.; Maksimovic, I.; Loriette, V.; Dubertret, B. Spectroscopy of Single CdSe Nanoplatelets. ACS Nano 2012, 6, 6751-6758.

(2) Wu, K.; Li, Q.; Jia, Y.; McBride, J. R.; Xie, Z.-X.; Lian, T. Efficient and ultrafast formation of long-lived charge-transfer exciton state in atomically thin cadmium selenide/cadmium telluride type-II heteronanosheets. ACS Nano 2015, 9, 961-968.

(3) Kelestemur, Y.; Guzelturk, B.; Erdem, O.; Olutas, M.; Gungor, K.; Demir, H. V. Platelet-in-Box Colloidal Quantum Wells: CdSe/ CdS@CdS Core/Crown@Shell Heteronanoplatelets. Adv. Funct. Mater. 2016, 26, 3570-3579.

(4) Luo, S.; Kazes, M.; Lin, H.; Oron, D. Strain-Induced Type II Band Alignment Control in CdSe Nanoplatelet/ZnS-Sensitized Solar Cells. J Phys Chem. C 2017, 121, 11136-11143.

(5) Sharma, M.; Gungor, K.; Yeltik, A.; Olutas, M.; Guzelturk, B.; Kelestemur, Y.; Erdem, T.; Delikanli, S.; McBride, J. R.; Demir, H. V. Near-Unity Emitting Copper-Doped Colloidal Semiconductor Quantum Wells for Luminescent Solar Concentrators. Adv. Mater. 2017, 29, No. 1700821.

(6) Guzelturk, B.; Kelestemur, Y.; Olutas, M.; Delikanli, S.; Demir, H. V. Amplified Spontaneous Emission and Lasing in Colloidal Nanoplatelets. ACS Nano 2014, 8, 6599-6605.

(7) Liu, B.; Sharma, M.; Yu, J.; Shendre, S.; Hettiarachchi, C.; Sharma, A.; Yeltik, A.; Wang, L.; Sun, H.; Dang, C.; Demir, H. V. Light-Emitting Diodes with Cu-Doped Colloidal Quantum Wells: From Ultrapure Green, Tunable Dual-Emission to White Light. Small 2019, 15, No. 1901983.

(8) She, C.; Fedin, I.; Dolzhnikov, D.; Dahlberg, P.; Engel, G.; Schaller, R.; Talapin, D. Red, Yellow, Green, and Blue Amplified Spontaneous Emission and Lasing Using Colloidal CdSe Nanoplatelets. ACS Nano 2015, 9, 9475-9485.

(9) Grim, J. Q.; Christodoulou, S.; Di Stasio, F.; Krahne, R.; Cingolani, R.; Manna, L.; Moreels, I. Continuous-wave biexciton lasing at room temperature using solution-processed quantum wells. Nat. Nanotechnol. 2014, 9, 891-895.

(10) Ithurria, S.; Bousquet, G.; Dubertret, B. Continuous Transition from $3 \mathrm{D}$ to $1 \mathrm{D}$ Confinement Observed during the Formation of CdSe Nanoplatelets. J. Am. Chem. Soc. 2011, 133, 3070-3077.

(11) Ithurria, S.; Dubertret, B. Quasi 2D Colloidal CdSe Platelets with Thicknesses Controlled at the Atomic Level. J. Am. Chem. Soc. 2008, 130, 16504-16505.

(12) Ithurria, S.; Talapin, D. V. Colloidal Atomic Layer Deposition (c-ALD) using Self-Limiting Reactions at Nanocrystal Surface Coupled to Phase Transfer between Polar and Nonpolar Media. J. Am. Chem. Soc. 2012, 134, 18585-18590.

(13) Fan, F.; Kanjanaboos, P.; Saravanapavanantham, M.; Beauregard, E.; Ingram, G.; Yassitepe, E.; Adachi, M.; Voznyy, O.; Johnston, A. K.; Walters, G.; Kim, G.; Lu, Z.; Sargent, E. Colloidal $\mathrm{CdSe}(1-\mathrm{x}) \mathrm{S}(\mathrm{x})$ Nanoplatelets with Narrow and ContinuouslyTunable Electroluminescence. Nano Lett. 2015, 15, 4611-4615.
(14) Sharma, M.; Delikanli, S.; Demir, H. V. Two-dimensional CdSe-based nanoplatelets: their heterostructures, doping, photophysical properties, and applications. Proc. IEEE 2020, 108, 655-675. (15) Khan, A. H.; Pinchetti, V.; Tanghe, I.; Dang, Z.; Martín-García, B.; Hens, Z.; Thourhout, D. V.; Geiregat, P.; Brovelli, S.; Iwan, M. Tunable and Efficient Red to Near-Infrared Photoluminescence by Synergistic Exploitation of Core and Surface Silver Doping of CdSe Nanoplatelets. Chem. Mater. 2019, 31, 1450-1459.

(16) Dufour, M.; Izquierdo, E.; Livache, C.; Martinez, B.; Silly Mathieu, G.; Pons, T.; Lhuillier, E.; Delerue, C.; Ithurria, S. Doping as a Strategy to Tune Color of 2D Colloidal Nanoplatelets. ACS Appl. Mater. Interfaces 2019, 11, 10128-10134.

(17) Qu, J.; Rastogi, P.; Gréboval, C.; Livache, C.; Dufour, M.; Chu, A.; Chee, S.; Ramade, J.; Zhen Xu, X.; Ithurria, S.; Lhuillier, E. Nanoplatelet-Based Light-Emitting Diode and Its Use in AllNanocrystal LiFi-like Communication. ACS Appl. Mater. Interfaces 2020, 12, 22058-22065.

(18) Altintas, Y.; Gungor, K.; Gao, Y.; Sak, M.; Quliyeva, U.; Bappi, G.; Mutlugun, E.; Sargent, E.; Demir, H. V. Giant Alloyed Hot Injection Shells Enable Ultralow Optical Gain Threshold in Colloidal Quantum Wells. ACS Nano 2019, 13, 10662-10670.

(19) Sharma, M.; Olutas, M.; Yeltik, A.; Kelestemur, Y.; Sharma, A.; Delikanli, S.; Guzelturk, B.; Gungor, K.; McBride, J. R.; Demir, H. V. Understanding the Journey of Dopant Copper Ions in Atomically Flat Colloidal Nanocrystals of CdSe Nanoplatelets Using Partial Cation Exchange Reactions. Chem. Mater. 2018, 30, 3265-3275.

(20) Sahu, A.; Kang, M.; Kompch, A.; Notthoff, C.; Wills, A.; Deng, D.; Norris, D.; et al. Electronic impurity doping in CdSe nanocrystals. Nano Lett. 2012, 2587-2594.

(21) Castro, N.; Bouet, C.; Ithurria, S.; Lequeux, N.; Constantin, D.; Levitz, P.; Pontoni, D.; Abécassis, B. Insights into the Formation Mechanism of CdSe Nanoplatelets Using in Situ X-ray Scattering. Nano Lett. 2019, 19, 6466-6474.

(22) She, C.; Fedin, I.; Dolzhnikov, D. S.; Dahlberg, P. D.; Engel, G. S.; Schaller, R. D.; Talapin, D. V. Red, Yellow, Green, and Blue Amplified Spontaneous Emission and Lasing Using Colloidal CdSe Nanoplatelets. ACS Nano 2015, 9, 9475-9485.

(23) Diroll, B. T.; Talapin, D. V.; Schaller, R. D. Violet-to-Blue Gain and Lasing from Colloidal CdS Nanoplatelets: Low-Threshold Stimulated Emission Despite Low Photoluminescence Quantum Yield. ACS Photonics 2017, 4, 576-583.

(24) Delikanli, S.; Isik, F.; Shabani, F.; Baruj, H. D.; Taghipour, N.; Demir, H. V. Ultralow Threshold Optical Gain Enabled by Quantum Rings of Inverted Type-I CdS/CdSe Core/Crown Nanoplatelets in the Blue. Adv. Opt. Mater. 2021, 9, No. 2002220.

(25) Di Giacomo, A.; Rodà, C.; Khan, A.; Moreels, I. Colloidal Synthesis of Laterally Confined Blue-Emitting 3.5 Monolayer CdSe Nanoplatelets. Chem. Mater. 2020, 32, 9260-9267.

(26) Vitukhnovsky, A. G.; Lebedev, V. S.; Selyukov, A. S.; Vashchenko, A. A.; Vasiliev, R. B.; Sokolikova, M. S. Electroluminescence of $\mathrm{CdSe} / \mathrm{CdS}$ quantum dots and the transfer of the exciton excitation energy in an organic light-emitting diode. Chem. Phys. Lett. 2015, 619, 185-188.

(27) Kelestemur, Y.; Dede, D.; Gungor, K.; Usanmaz, C. F.; Erdem, O.; Demir, H. V. Alloyed Heterostructures of CdSexS1-x Nanoplatelets with Highly Tunable Optical Gain Performance. Chem. Mater. 2017, 29, 4857-4865.

(28) Liu, B.; Altintas, Y.; Wang, L.; Shendre, S.; Sharma, M.; Demir, H. V.; et al. Record High External Quantum Efficiency of $19.2 \%$ Achieved in Light-Emitting Diodes of Colloidal Quantum Wells Enabled by Hot-Injection Shell Growth. Adv. Mater. 2019, No. 1905824.

(29) Zhang, F.; Wang, S.; Wang, L.; Lin, Q.; Shen, H.; Cao, W.; Yang, C.; Wang, H.; Yu, L.; Du, Z.; Xue, J.; Li, L. S. Super color purity green quantum dot light-emitting diodes fabricated by using CdSe/ CdS nanoplatelets. Nanoscale 2016, 8, 12182-12188.

(30) Hutter, E. M.; Bladt, E.; Goris, B.; Pietra, F.; van der Bok Johanna, C.; Boneschanscher Mark, P.; de Mello Donegá, C.; Bals, S.; Vanmaekelbergh, D. Conformal and Atomic Characterization of 
Ultrathin CdSe Platelets with a Helical Shape. Nano Lett. 2014, 14, 6257-6262.

(31) Tessier, M.; Spinicelli, P.; Dupont, D.; Gilles, G.; Ithurria, S.; Dubertret, B. Efficient Exciton Concentrators Built from Colloidal Core/Crown CdSe/CdS Semiconductor Nanoplatelets. Nano Lett. 2014, 14, 207-213.

(32) Ithurria, S.; Tessier, M. D.; Mahler, B.; Lobo, R. P.; Dubertret, S.; Efros, B. A. L. Colloidal nanoplatelets with two-dimensional electronic structure. Nat. Mater. 2011, 10, 936-941.

(33) Swafford, L. A.; Weigand, L. A.; Bower, M. J.; McBride, J. R.; Rapaport, J. L.; Watt, T. L.; Dixit, S. K.; Feldman, L. C.; Rosenthal, S. J. Homogeneously Alloyed CdSxSe1-x Nanocrystals: Synthesis, Characterization, and Composition/Size-Dependent Band Gap. J. Am. Chem. Soc. 2006, 128, 12299-12306.

(34) Maiti, P. S.; Houben, L.; Bar-Sadan, M. Growth Schemes of Tunable Ultrathin CdSXSe1 - X Alloyed Nanostructures at Low Temperatures. J. Phys. Chem. C 2015, 119, 10734-10739.

(35) Shendre, S.; Delikanli, S.; Mingiie, L.; Dede, D.; Pan, Z.; Tung Ha, S.; Fu, Y. H.; Hernández-Martínez, P.; Yu, J.; Erdem, O.; Kuznetsov, A. I.; Dang, C.; Sum, T. C.; Demir, H. V. Ultrahighefficiency aqueous flat nanocrystals of CdSe/CdS@Cd1-xZnxS colloidal core/crown@alloyed-shell quantum wells. Nanoscale 2019, 11, 301-310.

(36) Li, Z.; Qin, H.; Guzun, D.; Benamara, M.; Salamo, G.; Peng, X. Uniform thickness and colloidal-stable CdS quantum disks with tunable thickness: Synthesis and properties. Nano Res. 2012, 5, 337351.

(37) Di Giacomo, A.; Rodà, C.; Khan, A.; Moreels, I. Colloidal Synthesis of Laterally Confined Blue-Emitting 3.5 Monolayer CdSe Nanoplatelets. Chem. Mater. 2020, 32, 9260-9267.

(38) Wang, L.; Lin, J.; Hu, Y.; Guo, X.; Lv, Y.; Tang, Z.; Zhao, J.; Fan, Y.; Zhang, N.; Wang, Y.; Liu, X. Blue Quantum Dot LightEmitting Diodes with High Electroluminescent Efficiency. ACS Appl. Mater. Interfaces 2017, 9, 38755-38760.

(39) Xiao, P.; Huang, J.; Yan, D.; Luo, D.; Yuan, J.; Liu, B.; Liang, D. Emergence of Nanoplatelet Light-Emitting Diodes. Materials 2018, 11, No. 1376.

(40) Dai, X. L.; Zhang, Z. X.; Jin, Y. Z.; Niu, Y.; Cao, H. J.; Liang, X. Y.; Chen, L. W.; Wang, J. P.; Peng, X. G. Solution-processed, highperformance light-emitting diodes based on quantum dots. Nature 2014, 515, 96.

(41) Zhao, J.; Bardecker, J. A.; Munro, A. M.; Liu, M. S.; Niu, Y.; Ding, I. K.; Luo, J.; Chen, B.; Jen, A. K. Y.; Ginger, D. S. Efficient $\mathrm{CdSe} / \mathrm{CdS}$ quantum dot light-emitting diodes using a thermally polymerized hole transport layer. Nano Lett. 2006, 6, 463.

(42) Mahler, B.; Spinicelli, P.; Buil, S.; Quelin, X.; Hermier, J. P.; Dubertret, B. Towards non-blinking colloidal quantum dots. Nat. Mater. 2008, 7, 659.

(43) Shen, H.; Gao, Q.; Zhang, Y.; Lin, Y.; Lin, Q.; Li, Z.; Chen, L.; Zeng, Z.; Li, X.; Jia, Y.; Wang, S.; Du, Z.; Li, L.; Zhang, Z. Visible quantum dot light-emitting diodes with simultaneous high brightness and efficiency. Nat. Photonics 2019, 13, 192-197.

(44) Ji, W.; Wang, T.; Zhu, B.; Zhang, H.; Wang, R.; Zhang, D.; Chen, L.; Yang, Q.; Zhang, H. Highly efficient flexible quantum-dot light emitting diodes with an ITO/Ag/ITO cathode. J. Mater. Chem. C 2017, 5, 4543-4548.

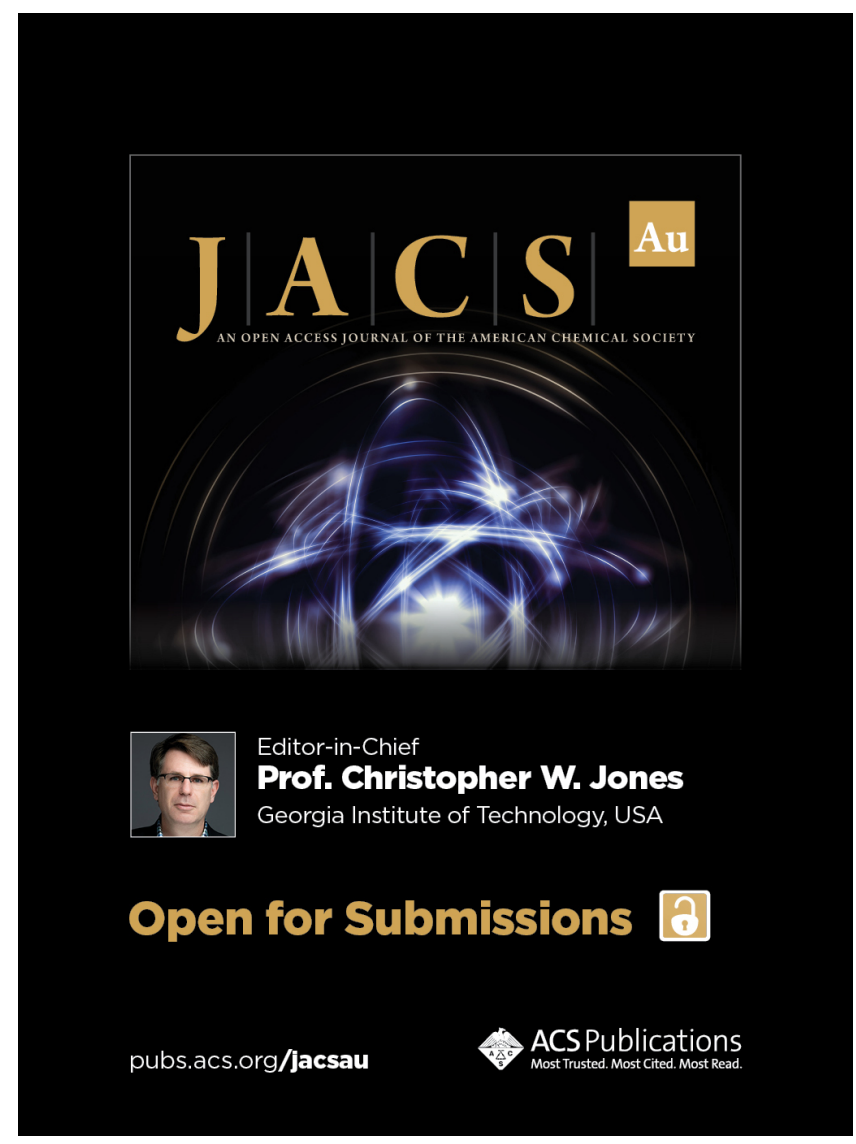

